\title{
A Multicenter Study of Neurocognition in Children with Hypertension: Methods, Challenges, and Solutions
}

\author{
Marc B. Lande, MD, MPH $\#$, Heather R. Adams, $\mathrm{PhD}^{\%}$, Juan C. Kupferman, MD, $\mathrm{MPH}^{\$}$, \\ Stephen R. Hooper, PhD, Peter G. Szilagyi, MD, MPH”, and Donald L. Batisky, MD@ \\ \#Department of Pediatrics, University of Rochester, Rochester, NY \\ \%Department of Neurology, University of Rochester, Rochester, NY \\ \$Department of Pediatrics, Maimonides Medical Center, Brooklyn, NY \\ Department of Pediatrics, University of North Carolina, Chapel Hill, NC \\ @Department of Pediatrics, Emory University, Atlanta, GA
}

\begin{abstract}
Hypertensive adults demonstrate decreased performance on neurocognitive testing compared with that of normotensive controls. There is now emerging, preliminary evidence that children with hypertension also manifest neurocognitive differences when compared to normotensive controls, findings postulated to potentially represent early signs of hypertensive target organ damage to the brain. However, reports in children to date have been limited to database and single-center studies. We have established an ongoing, prospective, multicenter study of neurocognition in children with primary hypertension. This article outlines the study methods, with particular attention to the unique challenges in this area of clinical research. We highlight aspects of the study design that are specifically designed as solutions to these challenges.
\end{abstract}

\section{Keywords}

Executive function; blood pressure; pediatric; cognition

\section{Introduction}

The prevalence of primary hypertension in children has increased significantly, a public health phenomenon that parallels the current childhood obesity epidemic. School screening studies show that the prevalence of hypertension is as high as $10 \%$ in children who are overweight, a remarkably high number given that nearly $20 \%$ of adolescents in the United States are obese.(1) The rising prevalence of pediatric hypertension raises concerns about impaired cardiovascular and other outcomes in adulthood.

\footnotetext{
(C) 2013 American Society of Hypertension. Published by Elsevier Inc. All rights reserved.

Corresponding Author: Marc B. Lande, MD, MPH, 601 Elmwood Ave., Box 777, Rochester, NY 14642, (Ph) 585-275-9784; (Fax) 585-756-8054, Marc_lande@urmc.rochester.edu.

None of the authors have any financial or other relations that could lead to a conflict of interest.

Publisher's Disclaimer: This is a PDF file of an unedited manuscript that has been accepted for publication. As a service to our customers we are providing this early version of the manuscript. The manuscript will undergo copyediting, typesetting, and review of the resulting proof before it is published in its final citable form. Please note that during the production process errors may be discovered which could affect the content, and all legal disclaimers that apply to the journal pertain.
} 
Recent studies suggest that hypertension during childhood may cause harm even during childhood.(2) Preliminary studies suggest that children with primary hypertension score lower on measures of neurocognition compared with normotensive controls. In particular, early findings suggest that hypertensive children have diminished scores in measures of executive function (EF; higher-order regulatory functions that include attention, inhibitory control, self-monitoring, planning/problem solving, working memory, and processing speed).(3,4), These findings are postulated to potentially represent an early manifestation of hypertensive target organ damage to the brain.(5) Further study of neurocognition in children with hypertension is needed to determine the impact of hypertension on the brain both in childhood and potentially later into adulthood, and to assess the degree to which any effects can be minimized, or perhaps, reversed.

With funding from the National Institutes of Health, we have established a prospective, multicenter study of neurocognition in children with primary hypertension. The objective of this article is to describe the study's methods, with particular emphasis on challenges and opportunities in designing studies to evaluate cognition in the setting of pediatric hypertension. This description of methods and challenges should provide guidance to researchers interested in this area of investigation.

\section{Study Description}

\section{Research Aims}

The specific aims of the study are to (1) define the impact of primary hypertension on executive function by comparing results of a comprehensive multimethod assessment of executive function in hypertensive subjects to results in normotensive controls, and (2) investigate the reversibility of executive dysfunction by evaluating the effect of antihypertensive therapy. In order to achieve these aims, both hypertensive and control subjects complete a baseline (pre-intervention) assessment consisting of laboratory evaluation and neurocognitive testing. The hypertensive subjects are then treated according to national consensus guidelines and local standards. After a 12-month period, the same neurocognitive testing is repeated in both the hypertensive and control subjects. Figure 1 shows the overall study design and Table 1 shows inclusion and exclusion criteria for hypertensive and normotensive groups.

\section{Sites and Participants}

Participating recruitment sites include the University of Rochester (PI, M. Lande), Emory University (PI, D. Batisky), and Maimonides Medical Center (PI, J. Kupferman). A total of seventy-five newly diagnosed, untreated hypertensive children aged $10-18$ years old will be recruited through the Pediatric Hypertension Clinics at each site. All hypertensive subjects are required to have clinic BP $\geq 95^{\text {th }}$ percentile and sustained hypertension confirmed by 24-hr ambulatory BP monitoring (ABPM). For comparison, 75 normotensive, healthy 10-18 year old children will be recruited across the three sites from participating general pediatric and family medicine practices. Control subjects are required to have clinic $\mathrm{BP}<95^{\text {th }}$ percentile and normotension confirmed by ABPM. The control group will be frequency matched (not individually matched) to the hypertensive group for maternal education, sex, and proportion with obesity (BMI $\geq 95^{\text {th }}$ percentile) with imbalances in other potential confounding variables adjusted for in the statistical analysis.

\section{Study Organization}

The University of Rochester Medical Center (URMC) serves as Clinical Coordinating Center (PI, M. Lande). Central laboratory tests are performed by the University of Rochester Clinical Trials Laboratory. The Steering Committee, comprised by each site PI, the Clinical 
Coordinating Center neuropsychologist (H. Adams), and the study senior neuropsychologist (S. R. Hooper), meets quarterly. The Safety Committee comprised by the URMC PI, the director of the Pediatric Clinical Research Office at URMC and the director of the Adult Hypertension Clinic at URMC meets at least twice a year and additionally as needed.

\section{Study Measures and Procedures}

ABPM-Study ABPM procedures follow published American Heart Association guidelines,(6) utilizing the Spacelabs 90217 oscillometric monitor. Blood pressure measurements are recorded every 20 minutes for the entire 24-hr period, and daytime and sleep periods are determined by patient diary. A minimum of 40 total readings, including at least 5 readings during sleep, are required for the study to be considered valid. BP load is defined as the percentage of readings above the $95^{\text {th }}$ percentile for ambulatory norms in the 24-hr period. For the hypertensive subjects to be included, they must have sustained ambulatory hypertension, defined as mean day or night SBP or DBP $\geq 95^{\text {th }}$ percentile for ambulatory norms. A hypertensive subject can also be included if the mean ambulatory BP is $<95^{\text {th }}$ percentile, but the subject has both BP load $>25 \%$ (ambulatory prehypertension) and left ventricular hypertrophy $(\mathrm{LVH})$ on echocardiogram. Control subjects are required to have both mean SBP and DBP $<95^{\text {th }}$ percentile and BP load $<25 \%$ to be included.

Echocardiography-Echocardiography is performed in the hypertensive subjects prior to the baseline visit and read centrally at the University of Rochester. Control subjects do not have echocardiography since they are not expected to have cardiac hypertrophy secondary to increased BP. Left ventricular mass indexed to height ${ }^{2.7}$ is determined by the area-length method according to standards published by the American Society of Echocardiography. Left ventricular hypertrophy is defined as left ventricular mass index (LVMI) $\geq$ the $95^{\text {th }}$ percentile for age and sex.(7)

Baseline Laboratory Evaluation-Both hypertensive and control subjects receive central laboratory evaluations including fasting lipid profile, insulin level, glucose and wide range CRP at the baseline assessment, due to the potential effects of hyperlipidemia,(8) insulin resistance,(9) and inflammation(10) on cognition.

Neurocognitive Test Battery-Previous studies show that both adult and pediatric hypertensive groups are particularly distinguished from non-hypertensive groups by performance on tasks of executive function (EF). Our pilot study also found decreased EF skills in hypertensive children, compared to normotensive controls, on parental behavior ratings of EF in everyday settings. $(5,8)$ Consequently, the current, expanded neurocognitive test battery was selected to evaluate EF in depth by including both performance-based laboratory measures and behavioral rating scales. The laboratory measures include direct tests of problem solving/planning, set-shifting, response inhibition, vigilance, and working memory, in order to assess components of EF defined in the Pennington and colleagues' theoretically-derived EF model (Table 2).(11,12) Defined globally as "the ability to maintain an appropriate problem solving set for attainment of a future goal,"(13) executive functions in Pennington's model incorporate 'process' skills such as self-monitoring/self-regulating behavior, set-shifting, planning, working memory, and processing speed, and also emphasize "context specific action selection"(11) which refers to the ability to appropriately select the correct strategy or response for the task at hand. The neurobiological substrate for this model is the prefrontal cortex which with its rich interconnections receives and integrates information from cortex, subcortex, and even brainstem sites. Since the neurocognitive test battery is not intended for clinical use, the test results are shared with parents only after the subject has completed the study, and only if specifically requested by the parent. 
Executive Function Assessment - laboratory measures: 1. CogState is a computerized battery of tests; $(14,15)$ selected EF measures from CogState are used in the present study. 1a. CogState Groton Maze Learning evaluates problem solving, cognitive flexibility, and nonverbal/visual learning. Subjects use trial-and-error strategies to learn a maze pattern over multiple learning trials. 1b.CogState Set Shifting is a task of concept formation and cognitive flexibility. Subjects must recognize and adapt to changing rule sets, using feedback from their own task performance to test and confirm hypotheses. 2. The Conners' Continuous Performance Test-II (CPT-II)(16) is a computerized test that provides measures of inattention, impulsivity, and response time. Subjects must respond when specific stimuli are presented on the computer screen, but inhibit this prepotent response when other stimuli appear. 3. Digit Span and Spatial Span (Wechsler Intelligence Scale for Children; WISC-IVIntegrated);(17) these tasks evaluate auditory and visual working memory, respectively. Subjects must repeat number sequences or copy manual sequences exactly; these are each followed by the more challenging working memory task of copying sequences in reverse order. 4. The Delis-Kaplan Executive Function System (D-KEFS) Tower Test is a measure of planning and problem-solving abilities.(18) Subjects must rearrange a stack of disks, in ascending size, based on models provided by the examiner. Movements are restricted along three pegs on which the disks are stacked, and specific rules limit subjects' movement of the disks from one peg to another (e.g., move only one piece at a time). The test assesses the subjects' ability to plan and execute an effective strategy in the shortest time, and with the fewest number of moves possible.

Executive Function Assessment - behavior rating scales: As noted above, the initial pilot study utilized the parent-report version of the Behavior Rating Inventory of Executive Function (BRIEF). The BRIEF evaluates EF within real-world settings.(19) The current study extends the assessment of EF in day-today life with addition of the self-report and teacher-based BRIEF ratings. This strategy yields multi-informant data, using crossvalidated measures, on subject's day-to-day EF behaviors such as organization and planning of homework and chores, the ability to sustain attention in class, or to navigate shifts between activities or cognitive tasks. BRIEF scores are norm-referenced to provide information on EF performance in comparison to same-age peers.

Assessment of other cognitive domains: In addition to EF measures, the neurocognitive test battery includes direct assessments of verbal learning (Rey Auditory Verbal Learning Test, RAVLT) and fine motor dexterity (Grooved Pegboard), based on prior evidence that children with hypertension may have diminished capacity in these cognitive domains in comparison to their normotensive peers. $(20,21)$ On the RAVLT, participants learn and recall a list of words presented over multiple learning trials.(22) Primary measures include list recall both in the short-term after the final learning trial, and again after a delay. The Grooved Pegboard test evaluates dominant and non-dominant hand fine-motor dexterity and speed.(22) Subjects use their fingertips to rapidly fit metal, "key"-shaped pegs into a matrix of holes. Finally, the direct neurocognitive evaluation includes an estimate of general intellectual function using the two-subtest version of the Wechsler Abbreviated Scales of Intelligence (WASI; Vocabulary, Matrix Reasoning).(23) This enables us to examine whether imbalances exist across hypertensive and normotensive groups with regard to global cognitive ability so as to lessen the effects of the level of cognitive ability on response to the antihypertensive treatment.

Results from our pilot studies show that children with both hypertension and obesity were at risk for clinically significant elevations in anxiety and depression symptoms.(3) The relationship between anxiety and hypertension has also long been reported in adult studies. (24) Therefore, in the current study, we continued to evaluate mood function in hypertensive children and the normotensive control group. In that regard, behavioral rating scales of 
mood state (anxiety, depression) and general behavioral function are administered. Mood symptoms are evaluated with the child self-report measures of the Multidimensional Anxiety Scale for Children (MASC),(25) and the Child Depression Inventory (CDI).(26) The Child Behavior Checklist (CBCL) provides additional parent-based assessment of the child's mood, and other behavioral concerns such as social difficulties, attention problems, noncompliance/defiance, and aggression.(27)

Because daytime sleepiness and sleep disorders are also associated with cognitive dysfunction in both adults and children, $(28,29)$ we also administer the Sleep-Related Breathing Disorder scale of the Pediatric Sleep Questionnaire (PSQ)(30). This survey is completed by parents to estimate disordered sleep overall; subjects are also asked to report number of hours of sleep they had received the night prior to neurocognitive testing as an estimate of sleepiness that day.

Standardized Antihypertensive Therapy-The study evaluates for changes in EF and related cognitive functions after 1 year of treatment that the hypertensive patient would normally receive according to local standard of care and national consensus guidelines.(2) Thus, the primary focus is on the effectiveness of intervention in a real-world setting; the efficacy of antihypertensive treatment is already well established in prior investigations.(31) For the hypertensive subjects, antihypertensive treatment (lifestyle modifications and/or medication) begins after completion of the baseline laboratory and neurocognitive assessments. For subjects with stage 1 hypertension without $\mathrm{LVH}$, antihypertensive medication is started after three months if there is no improvement with lifestyle modification, or is started immediately in subjects who have previously failed a concerted effort at lifestyle modification, at the discretion of the treating site investigator. Subjects with stage 2 hypertension or LVH are started immediately on antihypertensive medication. When antihypertensive medication is indicated, the initial drug is lisinopril. Angiotensin converting enzyme inhibitors are the most commonly prescribed class of antihypertensive medication prescribed by pediatric nephrologists for the treatment of primary hypertension, (32) and they are commonly used by the investigators at the participating sites. Female subjects of child bearing potential and their parents are counseled on the potential teratogenic risks of ACE inhibitors. Urine pregnancy tests are performed prior to initiating ACE inhibitor therapy and then every 3 months for the duration of the study. Blood chemistry measures for potassium and creatinine are performed within 4 weeks of starting lisinopril. Thiazide diuretics are used as second-line agents, if needed, except for competitive athletes, who receive amlodipine as a second-line agent. Amlodipine is also used for subjects who do not tolerate lisinopril.

Once antihypertensive medication is initiated, subjects are seen every $4-6$ weeks through the site's Pediatric Hypertension clinic to have their therapy titrated until adequate control of hypertension is achieved (casual BP $<95^{\text {th }}$ percentile). Initial dosing of antihypertensive medication and subsequent dose adjustments are at the discretion of the site investigator. Once adequate control is achieved, the hypertensive subjects are followed every 3 months to monitor BP control and adjust antihypertensive therapy accordingly. Subjects achieving BP control with life style modification alone are followed every 3 months throughout the course of the study. Upon enrollment, the subjects are given a home digital BP monitor (OMRON BP760) to measure their home BP once daily in the week prior to their next visit. The subjects keep a log of these measurements, which is then brought to all visits to help determine the response to therapy. All hypertensive subjects meet with a nutritionist at the beginning of the study and again at 3 - 6 months to review the Dietary Approaches to Stop Hypertension (DASH) diet,(33) salt restriction, and if needed, weight loss counseling. 


\section{Statistical Analyses}

The primary outcome is comparison of neurocognitive performance of hypertensives and controls at the baseline assessment. Seventy-five subjects per group provides $80 \%$ power to detect an effect size of 0.46 for baseline differences in the BRIEF Global Executive Composite, based on data from the pilot study.(3) Multivariate analyses will be adjusted for group imbalances in age, sex, maternal education, household income, race/ethnicity, IQ, obesity, hyperlipidemia, insulin resistance, inflammation, measures of anxiety and depression, disordered sleep, and hours of sleep the night prior to neurocognitive testing. In addition, all of the cognitive measures used are age-normed, providing standard metrics for evaluation of these data in relation to age-expected performance. Comparison of the hypertensive and control subjects at baseline will be done with and without the hypertensive subjects enrolled with ambulatory prehypertension but also LVH, since these subjects do not meet the American Heart Association definition of ambulatory hypertension.(6)

Performance of hypertensive subjects on neurocognitive testing after 1 year of antihypertensive therapy will be compared to their own baseline performance prior to antihypertensive therapy. Pre-post analysis will also be performed for the normotensive control subjects to estimate any practice effect or improvement with age. Within the hypertensive group, the correlation between the neurocognitive measures and systolic and diastolic ABPM parameters (including BP standard deviation as a measure of variability) will be determined. To further test the hypothesis that more severe hypertension is associated with greater reductions in cognitive function, we will also compare cognitive function between the hypertensive subjects with versus without LVH at the baseline assessment. Finally, among the hypertension subjects, the correlation between improvement in BP and improvement in neurocognition over the 12-month study period will be examined, adjusting for the duration of $\mathrm{BP}$ control $(<95$ th percentile) within the 12-month treatment period, estimated by interim clinic and home BP measurements.

\section{Challenges, Threats to Validity, and Solutions}

Challenge \#1 - Potential Confounders-Hypertensive-associated reductions in neurocognitive test performance tend to be relatively small in magnitude, and absolute scores fall within the broad developmentally normal range. $(5,8)$ Such findings may be overshadowed by subject characteristics that are known to more strongly influence performance on tests of cognition, such as socioeconomic status, depression, and anxiety.(8) In addition, a surprisingly high percentage of children referred for elevated BP have the diagnosis of a learning disability and/or attention deficit disorder,(34) both pathophysiologic processes that may strongly influence performance on neurocognitive testing and therefore overshadow any potential effect of hypertension. Furthermore, maternal education level can strongly influence performance on neurocognitive measures in children.(35) In addition, many children with hypertension are obese, an entity associated with metabolic syndrome, disordered sleep, and obstructive sleep apnea which are themselves associated with neurocognitive dysfunction. $(9,30)$ Finally, subject engagement with neurocognitive testing is essential. Critical neurocognitive test data can be lost when subjects are not well-rested on the day of examination, a particular challenge in studies involving adolescent participants. Table 3 lists the solutions incorporated into the study design in order to prevent these potential confounders from overshadowing any difference between groups attributable to hypertension.

Challenge \#2 - How to develop measurement for small effect sizes?-In order to detect the likely subtle between-group difference, the study ensures that the BP level of the groups is as distinct as possible by requiring ABPM confirmation of both hypertension and normotension. Individuals with pre-hypertension (except those with LVH) or white-coat 
hypertension by ABPM are excluded. Hypertension subjects will have had a variable number of elevated BP readings documented in the primary care office prior to referral. For initial consideration for the study, they are also required to have hypertension clinic BP $\geq 95^{\text {th }}$ percentile. By contrast, the control subjects are considered for recruitment for the study based on only one BP reading in the primary care office. However, the strict definition of ambulatory normotension, using both normal mean BP and also normal BP load should help prevent misclassification of BP category of the control subjects.

In addition, the neurocognitive test battery is designed to maximize the likelihood of detecting a relatively small effect size. Specifically, the CPT-II and CogState tasks, all computer-administered, enable precise measurement of response time, down to the millisecond, a feature that is especially desirable for evaluating potentially subtle differences between hypertensive and normotensive groups and also subtle changes within subjects over time. The computerized neurocognitive assessment data may detect small effects but have less relevance for every-day settings; therefore we have also included parent, child, and teacher-based questionnaires that evaluate executive function with high ecological validity (i.e., Behavior Rating Inventory for Executive Function).

Challenge \#3 - How to handle practice effects and developmental changes?Repeated administration of neurocognitive testing, such as before and after antihypertensive therapy, can be confounded by practice effects - the propensity for scores to improve by virtue of learned strategies or recall of task content from repeated test administration. This is a particularly common problem with repeat administration of standard tests of EF that often depend on novelty. Furthermore, test scores may improve with age, and repeated testing may yield improved scores due to regression to the mean. The study design incorporates several deliberate strategies to minimize the magnitude of practice effects and the effects of age:

1. All of the cognitive measures used are age-normed, providing standard metrics for evaluation of these data in relation to age-expected performance, and age is included as a covariate in the statistical analysis.

2. We wait a full 12 months to re-administer the test battery, thereby diminishing improvement due to retention of learned strategies or recall of task content.

3. CogState tasks were selected, in part, because the battery was designed for repeated assessments using alternate forms, thus minimizing practice effects.(36)

4. The use of a contemporaneous, well-matched control group that also completes reassessment after 12 months, permits estimation of, and adjustment for (if needed) potential practice effects and age-expected changes in test performance, independent of change attributable to antihypertensive therapy.

\section{Challenge \#4 - How to most ethically assess for the effect of antihypertensive treatment on cognition?-We have not proposed a randomized clinical trial in which} half of the hypertensive subjects receive antihypertensive medication and half receive placebo. While a randomized trial would have advantages, it would have meant excluding an essential group of subjects: those with stage 2 hypertension and those with LVH, since standard of care is to treat all such children with antihypertensive medication upfront.(2) In our pilot study, children with severe ambulatory hypertension and those with LVH at baseline were the subjects most likely to have improvement in executive function with antihypertensive therapy,(4) a finding that underscores the importance of not excluding these subjects from the current study. Furthermore, a randomized controlled trial would have meant giving placebo to some hypertensive children for a full year, even those children who have already made an earnest attempt at therapeutic lifestyle modification without success. 
While use of placebo is considered justifiable in pediatric antihypertensive drug trials, it is recommended that the duration of placebo use be limited to 4 to 8 weeks.(37) A randomized clinical trial with such a short duration would significantly increase the risk of practice effect on the neurocognitive measures and might not allow enough time for blood pressure control or cognitive improvement. Therefore, the reversibility of hypertension-associated neurocognitive deficits in the current trial is assessed by comparing each hypertensive subject to him or her self, before and after standard of care treatment.

An additional challenge is the inevitable variation in treatment compliance from subject-tosubject and also within the same subject over the course of a year. In the current study, the importance of adherence to antihypertensive medication and lifestyle modification is emphasized to the subject at each interim visit. Furthermore, to account for the variability in compliance and response to therapy, the statistical analysis will_adjust for the duration of BP control $\left(<95^{\text {th }}\right.$ percentile) within the 12-month treatment period, estimated by the interim clinic and home BP measurements.

Lastly, for patients treated with lisinopril, the current study design will not distinguish whether any improvement in cognition was due to the BP lowering effects of the lisinopril or to a direct effect of the drug itself. If improvement in cognition is demonstrated, then future research can compare the effect of different antihypertensive drug classes, including ACE inhibitors with differing levels of central nervous system penetration.(38)

Challenge \#5 - How to ascertain and maintain the matched sample?-The hypertensive subjects are recruited from referrals to the Pediatric Hypertension Clinics at each participating site. Control subjects are recruited from participating general pediatric and family medicine practices. Parents of potentially eligible control children are contacted with a letter sent by their primary care provider, and directed to contact the study coordinator if interested in the study. In order to ensure appropriate matching to the hypertensive group, the study coordinator assesses maternal education and screens for exclusion criteria by telephone, when contacted by the parents of prospective subjects. Potential control subjects who meet the targeted matching criteria (maternal education, obesity, age) are then enrolled. Site investigators are responsible for ongoing monitoring of the demographic characteristics of the enrolled subjects at their sites in order to ensure balance of matching criteria among hypertensive and control subjects. The PI and Steering Committee are responsible for oversight of monitoring of the demographics of enrolled subjects in the study as a whole. Demographic characteristics of enrolled hypertensive and control subjects are reviewed at each meeting of the Steering Committee so that adjustments can be made as needed to future enrollment targets in order to maintain balance of the matching criteria between groups. This also will help any future adjustments needed secondary to dropouts from the study and subject noncompliance.

In order to maximize retention of the study population, subjects receive reminder phone calls prior to each scheduled visit. Hypertensive subjects who miss treatment visits are contacted immediately to assure prompt rescheduling of the visit. Updated contact information is obtained during each follow-up visit for the hypertensive subjects and quarterly by phone for the control subjects. Lastly, subject honoraria are paid for both the baseline and 1-year neurocognitive assessment visits.

\section{Challenge \#6 - How to address issues of quality control and site communication?}

Quality control of the neurocognitive data: The neurocognitive test battery is administered by a child neuropsychologist or a graduate psychology trainee under direct supervision of a neuropsychologist at each site. All neuropsychological examiners are 
blinded to the subject's BP status. The URMC clinical coordinating center neuropsychologist (H Adams) reviews the first 5 cases completed from each participating site and then subsequently reviews a random selection of $20 \%$ of the cases from all 3 sites. These audits are conducted to verify administration fidelity and to perform double scoring of the protocol procedures. Double scoring ensure that raw score points are accurately awarded and are accurately transformed into age-adjusted standardized scores, and that no transcription errors occur from source documents to study case report forms. The senior study neuropsychologist (S Hooper) will review a random selection of $20 \%$ of the cases administered by the URMC neuropsychologist.

Quality control of the treatment protocol: The decision to initiate antihypertensive medication and timing and magnitude of dosing adjustments is entrusted to the treating site investigator, within the general guidelines of the study protocol with regard to choice of medication (first line, lisinopril), frequency of follow up visits, and treatment goals (clinic $\mathrm{BP}<95^{\text {th }}$ percentile). To monitor adherence to these general guidelines, the clinical coordinating center monitors the frequency of interim treatment visits at the participating sites. Home and interim visit BP measurements and antihypertensive medication adjustments are reported to the clinical coordinating center after each interim visit.

Site communication: The clinical coordinating center study coordinators and PI are available to the participating sites on a daily basis, as needed. Conference calls between the clinical coordinating center and the individual participating sites take place at least monthly, in order to review recruitment progress, study procedures, and any problems as they arise. Annual meetings of the study personnel occur at the Coordinating Center.

\section{Summary}

The main objectives of this ongoing, multi-center study are to determine the impact of primary hypertension on neurocognition in children and whether these effects are reversible with treatment of hypertension. In this technical review, we have summarized the design and methods of the study, and shared the unique challenges that exist in assessing subtle changes in neurocognition, reflecting potential hypertension-associated target organ damage of the pediatric brain; we have also discussed real, concrete solutions to these unique challenges that are applicable to the clinicians, teacher, and researcher.

Translational studies such as this are critical to determine what BP levels result in brain target organ effects, both in childhood and adolescence and potentially later into adulthood. Clarification of the detrimental effects of hypertension during childhood will help in the development of improved treatment guidelines targeted at the children most at risk for poor cardiovascular outcomes later in life. It is essential for those engaged with this population to pay careful attention to the myriad of challenges that could affect the results of a treatment trial as these challenges can affect the care of children with hypertension.

\section{Acknowledgments}

We thank Jennifer Staskiewicz, Jill Casey, Kara Paterno, Margret Kamel, and Irena Kizer for excellent study coordination. We thank Drs. Natalie Arnette and Lisa Altshuler for all their efforts with the neurocognitive assessments.

Funding: This study is funded by NHLBI grant R01 HL098332 (PI, M. Lande). 


\section{References}

1. McNiece KL, Poffenbarger TS, Turner JL, Franco KD, Sorof JM, Portman RJ. Prevalence of hypertension and pre-hypertension among adolescents. J Pediatr. 2007 Jun; 150(6):640-4. 644.e1. [PubMed: 17517252]

2. National High Blood Pressure Education Program Working Group on High Blood Pressure in Children and Adolescents. The fourth report on the diagnosis, evaluation, and treatment of high blood pressure in children and adolescents. Pediatrics. 2004 Aug; 114(2 Suppl 4th Report):555-576. [PubMed: 15286277]

3. Lande MB, Adams H, Falkner B, Waldstein SR, Schwartz GJ, Szilagyi PG, et al. Parental Assessments of Internalizing and Externalizing Behavior and Executive Function in Children with Primary Hypertension. J Pediatr. 2009; 154(2):207-212. [PubMed: 18823913]

4. Lande MB, Adams H, Falkner B, Waldstein SR, Schwartz GJ, Szilagyi PG, et al. Parental assessment of executive function and internalizing and externalizing behavior in primary hypertension after anti-hypertensive therapy. J Pediatr. 2010 Jul; 157(1):114-119. [PubMed: 20227722]

5. Lande MB, Kupferman JC, Adams HR. Neurocognitive alterations in hypertensive children and adolescents. J Clin Hypertens (Greenwich). 2012 Jun; 14(6):353-359. [PubMed: 22672088]

6. Urbina E, Alpert B, Flynn J, Hayman L, Harshfield GA, Jacobson M, Mahoney L, McCrindle B, Mietus-Snyder M, Steinberger J, Daniels S. American Heart Association Atherosclerosis, Hypertension, and Obesity in Youth Committee. Ambulatory blood pressure monitoring in children and adolescents: recommendations for standard assessment: a scientific statement from the American Heart Association Atherosclerosis, Hypertension, and Obesity in Youth Committee of the council on cardiovascular disease in the young and the council for high blood pressure research. Hypertension. 2008 Sep; 52(3):433-451. [PubMed: 18678786]

7. Khoury PR, Mitsnefes M, Daniels SR, Kimball TR. Age-specific reference intervals for indexed left ventricular mass in children. Journal of the American Society of Echocardiography. 2009 Jun; 22(6):709-714. [PubMed: 19423289]

8. Waldstein, SR.; Snow, J.; Muldoon, MF.; Katzel, LI. Neuropsychological consequences of cardiovascular disease. In: Tarter, RE.; Butters, M.; Beers, SR., editors. Medical Neuropsychology. 2nd. New York: Kluwer Academic/Plenum Publishers; 2001. p. 51-51.p. 83

9. Yau PL, Castro MG, Tagani A, Tsui WH, Convit A. Obesity and metabolic syndrome and functional and structural brain impairments in adolescence. Pediatrics. 2012 Oct; 130(4):e856-64. [PubMed: 22945407]

10. Marsland AL, Petersen KL, Sathanoori R, Muldoon MF, Neumann SA, Ryan C, et al. Interleukin-6 covaries inversely with cognitive performance among middle-aged community volunteers. Psychosom Med. 2006 Nov-Dec;68(6):895-903. [PubMed: 17132839]

11. Pennington BF, Ozonoff S. Executive functions and developmental psychopathology. J Child Psychol Psychiatry. 1996 Jan; 37(1):51-87. [PubMed: 8655658]

12. Willcutt EG, Doyle AE, Nigg JT, Faraone SV, Pennington BF. Validity of the executive function theory of attention-deficit/hyperactivity disorder: a meta-analytic review. Biol Psychiatry. 2005 Jun 1; 57(11):1336-1346. [PubMed: 15950006]

13. Welsh MC, Pennington BF. Assessing frontal lobe functioning in children: Views from developmental psychology. Developmental neuropsychology. 1988; 4:199-230.

14. Pietrzak RH, Maruff P, Mayes LC, Roman SA, Sosa JA, Snyder PJ. An examination of the construct validity and factor structure of the Groton Maze Learning Test, a new measure of spatial working memory, learning efficiency, and error monitoring. Arch Clin Neuropsychol. 2008 Jul; 23(4):433-445. [PubMed: 18448309]

15. Falleti MG, Maruff P, Collie A, Darby DG. Practice effects associated with the repeated assessment of cognitive function using the CogState battery at 10-minute, one week and one month test-retest intervals. J Clin Exp Neuropsychol. 2006 Oct; 28(7):1095-1112. [PubMed: 16840238]

16. Conners, CK.; Staff, MHS. Computer program for Windows technical guide and software manual. North Tonawanda, NY: Mulit-Health Systems, Inc.; 2001. Conners' Continuous Performance Test II. 
17. Wechsler, D. Wechsler Intelligence Scale for Children- Fourth Edition Integrated. San Antonio, TX: The Psychological Corporation; 2004.

18. Delis, DC.; Kaplan, E.; Kramer, JH. Delis-Kaplin executive function system. San Antonio, TX: The Psychological Corporation; 2001.

19. Gioia, GA.; Isquith, PK.; Guy, SC.; Kenworthy, L. Behavior rating inventory of executive function. Lutz, FL: Pyschological Assessments Resources; 2000.

20. Lande MB, Adams H, Ogawa M, Pressler ML, McLain R, Palumbo D. Cognitive function in a 1year, open-label study of the safety of eplerenone in children. J Clin Hypertens. 2008; (10) (5):A154.

21. Lande MB, Schwartz GJ, Falkner B, Szilagyi PG, Adams H, Waldstein SR, et al. Treatment of pediatric primary hypertension is associated with improvement in neurocognitive function. J Clin Hypertens. 2008; (10)(5):A3.

22. Lezak, MD.; Howieson, DB.; Loring, DW. Neuropsychological Assessment. Fourth. New York: Oxford University Press; 2004.

23. Psychological Corporation. Wechsler Abbreviated Scale of Intelligence Manual. San Antonio, TX: The Pschological Corporation; 1999.

24. Jonas BS, Lando JF. Negative affect as a prospective risk factor for hypertension. Psychosom Med. 2000 Mar-Apr;62(2):188-196. [PubMed: 10772396]

25. March, JS. Manual for the Multidimentional Anxiety Scale for Children (MASC). Toronto, ON: Multi-Health Systems; 1997.

26. Kovacs, M. Children's Depression Inventory Manual. North Tonawanda, NY: Multi-Health Systems; 1992.

27. Achenbach, TM.; Rescorla, LA. Manual of the ASEBA school-aged forms and profiles. Burlington, VT: Research Center for Children, Youth, and Families; 2001.

28. Wolfson AR, Carskadon MA. Sleep schedules and daytime functioning in adolescents. Child Dev. 1998 Aug; 69(4):875-887. [PubMed: 9768476]

29. Durmer JS, Dinges DF. Neurocognitive consequences of sleep deprivation. Semin Neurol. 2005 Mar; 25(1):117-129. [PubMed: 15798944]

30. Chervin RD, Weatherly RA, Garetz SL, Ruzicka DL, Giordani BJ, Hodges EK, et al. Pediatric sleep questionnaire: prediction of sleep apnea and outcomes. Archives of Otolaryngology -- Head \& Neck Surgery. 2007 Mar; 133(3):216-222. [PubMed: 17372077]

31. Flynn JT, Daniels SR. Pharmacologic treatment of hypertension in children and adolescents. J Pediatr. 2006 Dec; 149(6):746-754. [PubMed: 17137886]

32. Woroniecki RP, Flynn JT. How are hypertensive children evaluated and managed? A survey of North American pediatric nephrologists. Pediatr Nephrol. 2005 Jun; 20(6):791-797. [PubMed: 15809834]

33. Couch SC, Saelens BE, Levin L, Dart K, Falciglia G, Daniels SR. The efficacy of a clinic-based behavioral nutrition intervention emphasizing a DASH-type diet for adolescents with elevated blood pressure. J Pediatr. 2008 Apr; 152(4):494-501. [PubMed: 18346503]

34. Adams HR, Szilagyi PG, Gebhardt L, Lande MB. Learning and attention problems among children with pediatric primary hypertension. Pediatrics. 2010 Dec; 126(6):e1425-9. [PubMed: 21059718]

35. Bornstein, MH.; Bradley, RF., editors. Socioeconomic Status, Parenting, and Child Development. Mahwuh, NJ: Erlbaum; 2003.

36. Falleti MG, Maruff P, Collie A, Darby DG. Practice effects associated with the repeated assessment of cognitive function using the CogState battery at 10-minute, one week and one month test-retest intervals. J Clin Exp Neuropsychol. 2006 Oct; 28(7):1095-1112. [PubMed: 16840238]

37. Flynn JT. Ethics of placebo use in pediatric clinical trials: the case of antihypertensive drug studies. Hypertension. 2003 Nov; 42(5):865-869. [PubMed: 14504262]

38. Sink KM, Leng X, Williamson J, Kritchevsky SB, Yaffe K, Kuller L, et al. Angiotensin-converting enzyme inhibitors and cognitive decline in older adults with hypertension: results from the Cardiovascular Health Study. Arch Intern Med. 2009 Jul 13; 169(13):1195-1202. [PubMed: 19597068] 


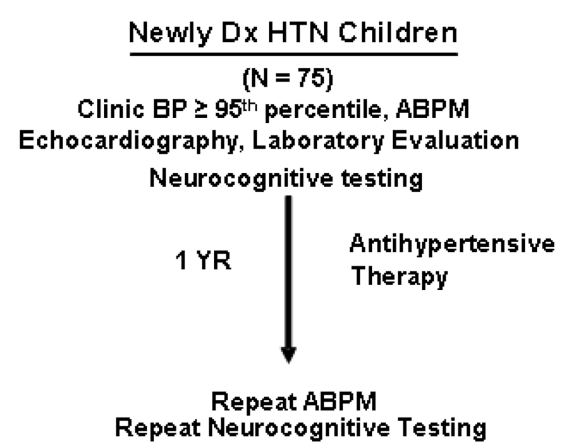

Repeat Neurocognitive Testing

\section{Normal BP Controls}

$(\mathrm{N}=75)$

Clinic BP $<95^{\text {th }}$ percentile, ABPM

Laboratory Evaluation

Neurocognitive testing

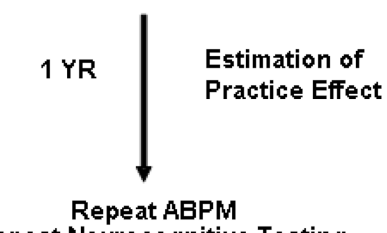

Repeat Neurocognitive Testing

Figure 1. Study design 


\section{Table 1}

\section{Inclusion and exclusion criteria}

Inclusion

Age 10 to $18 \mathrm{y}$

Primary hypertension

English speaking (required for the neurocognitive assessments)

Clinic SBP and/or DBP $\geq 95^{\text {th }}$ percentile

Confirmed hypertension (hypertension subjects) or normotension (control subjects) by 24-hr ABPM

Informed consent

Exclusion

Secondary hypertension

Previous or current treatment with antihypertensive medication

Learning disability (Individual Education Plan or Section 504 Plan at school)

Treatment with medication for inattention

Disorder of cognitive function, seizure disorder, or history of severe head injury (loss of consciousness $>30 \mathrm{~min}$ ) History of chronic disease

Previous chelation treatment for elevated lead level

Previous sleep study diagnosis of obstructive sleep apnea

Pregnancy or breastfeeding 


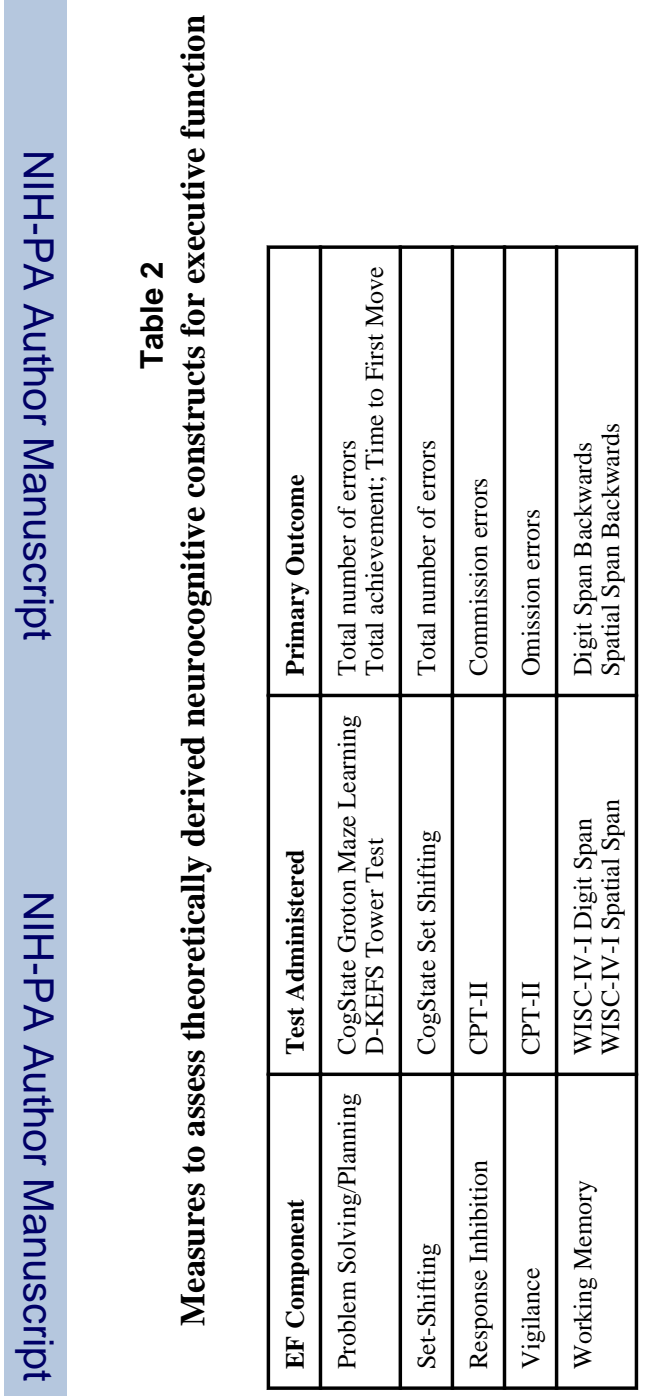

J Am Soc Hypertens. Author manuscript; available in PMC 2014 September 01. 


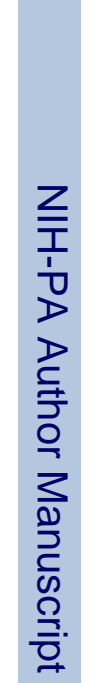

\begin{tabular}{|c|c|c|c|c|c|c|}
\hline 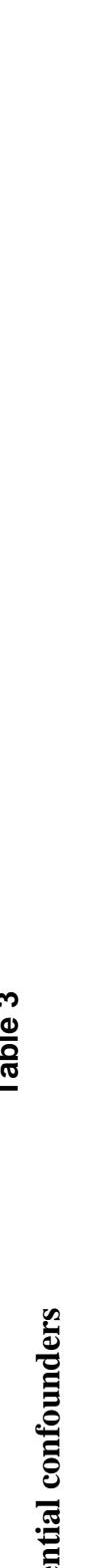 & 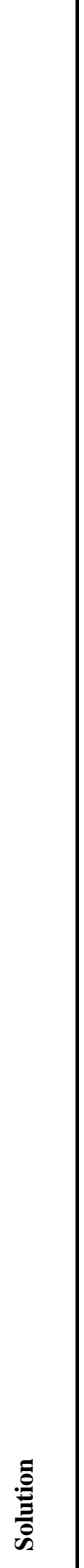 & 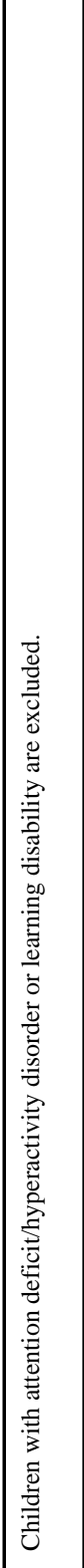 & 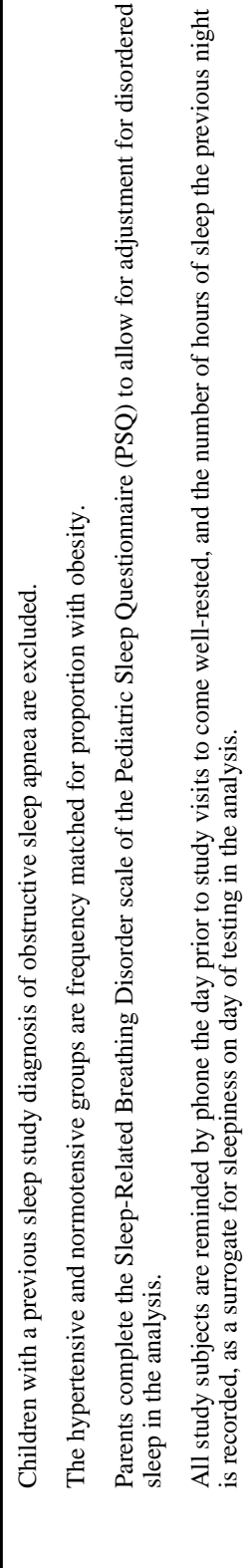 & 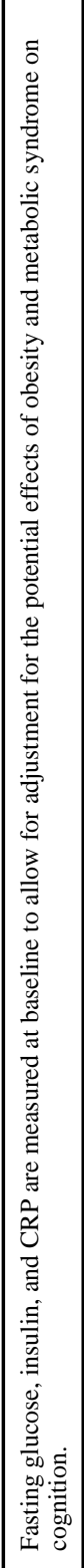 & 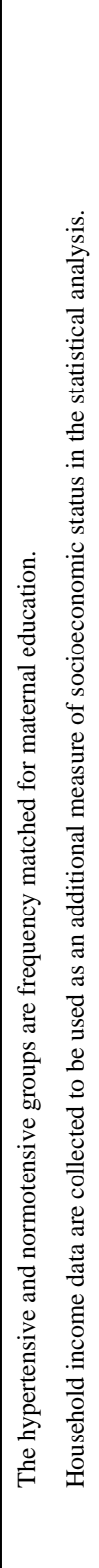 & 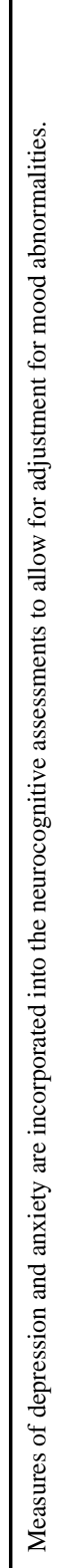 \\
\hline 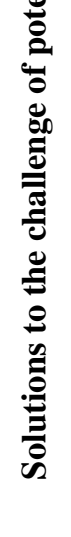 & 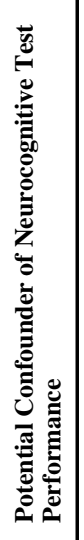 & 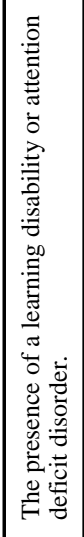 & 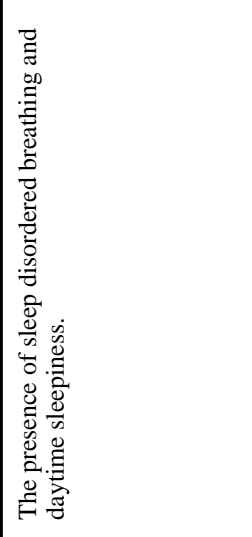 & 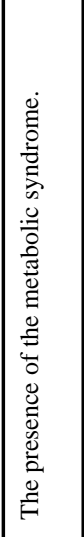 & 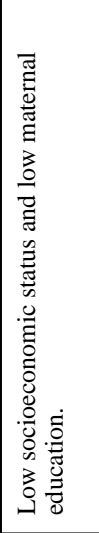 & 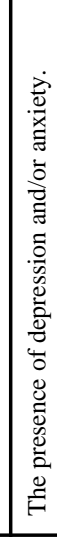 \\
\hline
\end{tabular}

J Am Soc Hypertens. Author manuscript; available in PMC 2014 September 01. 